The Influence of Vortex Pinning and Grain Boundary Structure on Critical Currents across Grain Boundaries in $\mathrm{YBa}_{2} \mathrm{Cu}_{3} \mathrm{O}_{x}$

Dean J. Miller, Kenneth E. Gray, Michael B. Field Materials Science Division, Argonne National Laboratory, Argonne, IL 60439

And

D.H. Kim

Department of Physics, Yeungnam University, Kyungsan 712-749, Korea

The submitted manuscripl has been crealed by the University of Chicago as Operator of Argonne National Laboratory ("Argonne") under Contract No. W-31-109-ENG-38 with the U.S. Department of Energy. The U.S. Government retains for itsell, and others acting on its behalf, a paid-up. nonexclusive. irrevocable worldwide license in said anticle itrevocable worldwide license in said anicle tribute copies to the public, and perform publicly and display publicly, by or on behalf of the Government.

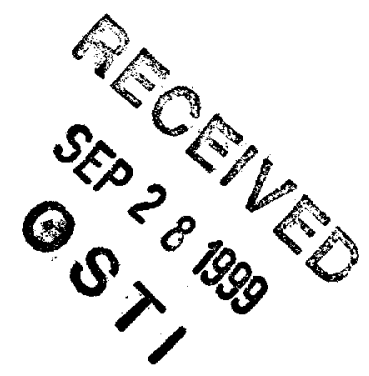

Proceedings of the 1998 Applied Superconductivity Conference, Palm Desert, CA, September 13-18, 1998, to be published in IEEE Transactions.

This work supported by the U.S. Department of Energy, Basic Energy Sciences-Material Sciences and Energy Efficiency and Renewable Energy under contract \#W-31-109-ENG-38 and National Science Foundation, Office of Science and Technology Centers for Superconductivity under contract No. DMR91-20000. 


\section{DISCLAIMER}

This report was prepared as an account of work sponsored by an agency of the United States Government. Neither the United States Government nor any agency thereof, nor any of their employees, make any warranty, express or implied, or assumes any legal liability or responsibility for the accuracy, completeness, or usefulness of any information, apparatus, product, or process disclosed, or represents that its use would not infringe privately owned rights. Reference herein to any specific commercial product, process, or service by trade name, trademark, manufacturer, or otherwise does not necessarily constitute or imply its endorsement, recommendation, or favoring by the United States Government or any agency thereof. The views and opinions of authors expressed herein do not necessarily state or reflect those of the United States Government or any agency thereof. 


\section{DISCLAIMER}

Portions of this document may be illegible in electronic image products. Images are produced from the best available original document. 


\title{
The Influence of Vortex Pinning and Grain Boundary Structure on Critical Currents across Grain Boundaries in $\mathrm{YBa}_{2} \mathrm{Cu}_{3} \mathrm{O}_{\mathbf{x}}$
}

\author{
Dean J. Miller, Kenneth E. Gray, Michael B. Field \\ Materials Science Division, Argonne National Laboratory, Argonne, IL 60439 \\ DongHo Kim \\ Department of Physics, Yeungnam University, Kyungsan 712-749, Korea
}

\begin{abstract}
We have used studies of single grain boundaries in YBCO thin films and bulk bicrystals to study the influence of vortex pinning along a grain boundary on dissipation. The critical current density for transport across grain boundaries in thin films is typically more than an order of magnitude larger than that measured for transport across grain boundaries in bulk samples. For low misorientation angles, the difference in critical current density within the grains that form the boundary can contribute to the substantial differences in current density measured across the boundary. However, substantial differences exist in the critical current density across boundaries in thin film compared to bulk bicrystals even in the higher angle regime in which grain boundary dissipation dominates. The differences in critical current density in this regime can be understood on the basis of vortex pinning along the boundary.
\end{abstract}

\section{I.INTRODUCTION}

The critical current across grain boundaries in $\mathrm{YBa}_{2} \mathrm{Cu}_{3} \mathrm{O}_{x}$ (YBCO) has been measured in a large number of studies using a variety of types of samples since Dimos, et al.[1] first demonstrated the "weak link" effect of grain boundaries in these materials. Through all of these studies, it has been clearly shown that the critical current carried across a grain boundary is a strong function of the misorientation angle between the grains that form the boundary. A variety of models have been proposed that attempt to explain the fundamental dependence of critical current on misorientation angle.[2-5] However, the critical current $\left(I_{c}\right)$ and critical current density $\left(\mathrm{J}_{c}\right)$ of a grain boundary are macroscopic quantities that reflect not only the intrinsic coupling across the boundary (which would determine electron conduction) but also external parameters, such as flux motion along the boundary (which contributes to measured dissipation). Thus, both factors are important in determining the transport properties of a grain boundary.

An illustration of the difficulty in isolating the key factors that result in reduced critical currents across grain boundaries in high temperature superconductors is shown in Fig. 1. In this figure, previously reported [6-8] results of $J_{c}$ measured across single grain boundaries in thin film bicrystals and in bulk bicrystals is plotted as a function of the misorientation

Manuscript received September 14, 1998.

This work as supported by National Science Foundation Office of Science and Technology Centers (\#DMR 91-20000) and by the U.S. Department of Energy, Basic Energy Sciences-Materials Sciences, and Energy Efficiency and Renewable Energy (\#W-31-109-ENG-38). angle, $\theta$, between the grains. Qualitatively, the data for the thin film and bulk bicrystal grain boundaries is similar: each shows a sharp decrease in $J_{c}$ across the boundary as $\theta$ increases. However, there are significant differences in the data when viewed quantitatively. Firstly, the absolute magnitude of $J_{c}$ measured for grain boundaries in the bulk samples is significantly lower than those measured for the thin film counterparts. Secondly, the functional dependence of $J_{c}$ on $\theta$ is weaker in the case of the bulk samples compared to the thin films, with the slope of $J_{c}(\theta)$ for the bulk samples approximately half of that for the thin films. Recently, Gray, et al.[9] have proposed a model that considers the pinning of vortices along the grain boundary to explain the discrepancies in the data of Fig. 1. In this paper, we examine several of the key consequences of this model and compare the predictions based on them with experimental data. We find that pinning of vortices along a grain boundary can have a substantial impact on the $J_{c}$ measured across the boundary, suggesting that a complete picture of current transport across grain boundaries must include consideration of vortex dynamics and the influence of vortex motion on measured dissipation.

\section{II.EXPERIMENTAL}

Bicrystals of YBCO were prepared for this work using a dual-seeded melt textured growth process that has been described fully elsewhere. [8] In short, two $\mathrm{NdBa}_{2} \mathrm{Cu}_{3} \mathrm{O}_{x}$ seed crystals are used to nucleate the growth of two domains in a YBCO pellet when subjected to a melt-texturing process, resulting in a bicrystal with the misorientation angle and axis determined by the seeding method. The YBCO pellet contains $\mathrm{YBCO}$ plus about 25 vol. $\% \mathrm{Y}_{2} \mathrm{BaCuO}_{\mathrm{x}}$ (211), so that the YBCO grains in the bicrystal also contain small 211 particles. Transmission electron microscopy (TEM) has shown that the 211 is uniformly distributed throughout the sample and is not segregated to the boundary. Thin film bicrystals for these studies were prepared by off-axis sputter deposition onto commercially available $\mathrm{SrTiO}_{3}$ bicrystal substrates so that the misorientation angle and axis in the YBCO bicrystal is determined by the substrate.

Transport measurements were carried out using a 4- or 6terminal configuration at a temperature of $77 \mathrm{~K}$. Magnetic fields up to $0.1 \mathrm{~T}$ were also applied. In most cases, the field was applied with $\mathrm{H}$ parallel to the $c$-axes of the grains. The dependence of $J_{c}$ on field orientation was measured for one $90^{\circ}[100]$ tilt boundary by rotating the field in the plane of the boundary. 


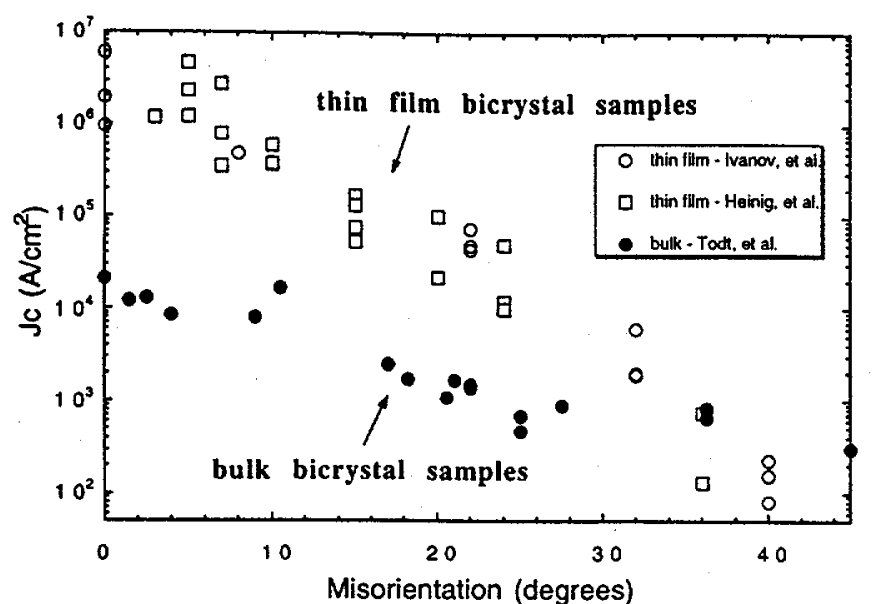

Figure 1. Critical current density $(\mathrm{Jc})$ at $77 \mathrm{~K}, \mathrm{~B}=0$, as a function of misorientation angle, $\theta$, for single grain boundaries in thin film and bulk bicrystals. The data from Ivanov [6], Heinig [7], and Todt [8] are all for nominal [001] tilt boundaries.

\section{III.RESULTS}

As noted above for Fig. 1, the differences in $J_{c}$ measured across grain boundaries in bulk bicrystals are significantly lower than those measured for the thin film counterparts. In the very low angle regime $\left(\theta\right.$ less than $\approx 10^{\circ}$ ), the difference may be ascribed to the significantly lower $J_{c}$ in the grains on each side of the boundary in the bulk samples compared to relatively high $J_{c}$ values typical of high quality thin films. Since each transport measurement of a grain boundary includes some contribution from the grains on each side due to the placement of voltage contacts, any dissipation in the grains contributes to the measured voltage. As a result, the values measured for the grain boundary $J_{c}$ in the bulk samples are influenced by the $J_{c}$ for the grains (to a degree influenced by the spacing of the voltage taps for an electric field criterion) and should in fact be somewhat lower if the boundary also contributes to the dissipation as well. However, in the higher angle regime, the $J_{c}$ values clearly reflect dissipation due to the grain boundary in both the thin film and bulk bicrystal cases. For example, looking at the values for $\theta \approx 20^{\circ}$, it is seen that in both cases the $J_{c}$ values measured are nearly an order of magnitude lower than the low $\theta$ values, suggesting the strong influence of the boundary. In this situation, it is difficult to explain the difference in critical current between the two types of boundaries which are nominally identical.

Although all of the data of Fig. 1 is for boundaries that are nominally [001] tilt boundaries, it has been shown that the boundaries in thin film bicrystals are not well defined but instead meander significantly, leading to a grain boundary plane that changes as a function of position along the boundary. In contrast, the grain boundaries in the bulk bicrystals have been shown to be highly planar over a wide range of length scales. An example of the typical microstructures for each type of boundary is shown in Fig. 2. Based on this structural difference, Gray, et al.[9] proposed a model based on the pinning of Josephson vortices along the boundary to explain the discrepancy in $J_{c}$ values. In this model, it is assumed that the highly meandering grain configuration of thin film grain boundaries is effective in pinning a Josephson vortex while the smooth, planar configuration of the bulk boundaries would pose little resistance to motion. The presence of Josephson vortices is assumed as a consequence of the weak coupling across the boundaries, and the Josephson critical current, $J_{\mathrm{cJ}}$, follows an exponential decay with the misorientation angle, $\theta$. Under these circumstances, the $J_{c}$ measured for the thin film boundaries should reflect the degree of pinning and scale linearly with $J_{c J}$. In the case of the bulk bicrystals, $J_{c}$ occurs once a vortex enters the boundary since it may then be driven along the boundary rather freely, leading to dissipation. In this situation, $J_{c}$ is expected to scale as $J_{c j}{ }^{1 / 2}$. Based on this model, the difference in the magnitude of $J_{c}$ as well as the difference in the slope of $J_{c}(\theta)$ in Fig. 1 can be accurately predicted.

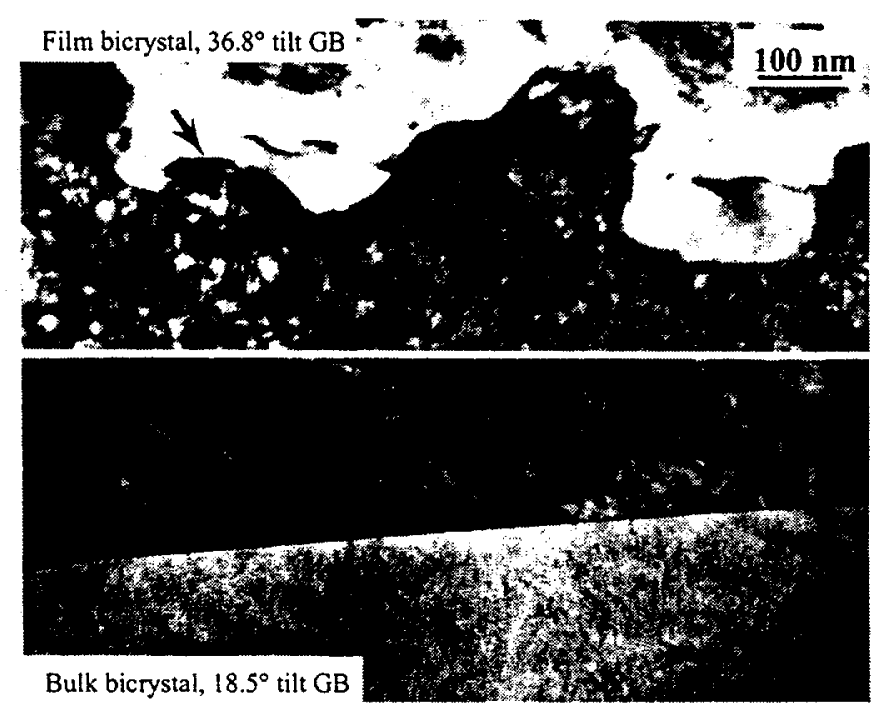

Figure 2. Bright-field TEM micrographs showing the typical microstructure of a thin film [001] tilt grain boundary (top) and a bulk [001] tilt boundary.

In evaluating this model, one must consider whether the assumption that pinning of Josephson vortices based on the meandering configuration of the boundary is reasonable. The dominant contribution to the energy of a Josephson vortex is associated with the circulating screening currents. Intuitively, any redistribution of the currents required to move the vortex would be resisted. Thus, the geometrical deviations of the boundary are expected to promote pinning. In addition, the grain boundary plane also changes as a function of position, and it has been speculated that the strength of coupling may be a function of the grain boundary plane. Recently, we have been able to measure the effect of the boundary plane on $J_{c}$ using $90^{\circ}$ [100] grain boundaries. [10] In these measurements, we have found a substantial difference in $\mathrm{J}_{\mathrm{c}}$ based on the boundary plane, with $90^{\circ}$ [100] twist boundaries supporting a $\mathrm{J}_{\mathrm{c}}$ nearly an order of magnitude higher than their $90^{\circ}$ [100] symmetric tilt counterpart. Thus, the idea that pinning of Josephson vortices in the thin films boundaries can be strong is reasonable based on a boundary consisting of a series of different planes. 
In contrast to the thin films, microstructural characterization of the bulk boundaries reveals no features that would be effective in pinning vortices. Under these circumstances, $J_{c}$ is determined once the magnetic field reaches $\mathrm{H}_{\mathrm{cl}}$ and vortices can penetrate the boundary. A consequence of this factor is that $I_{c}$ should scale inversely with applied magnetic field since the total field is equal to the applied field plus the self-field generated as a result of the current passing through the sample. Figure 3 shows data of $I_{c}$ as a function of applied field for two different bulk grain boundaries. In each case, the initial decrease obeys the expected relationship. Note that $I_{c}(0)$ for the $25^{\circ}$ boundary is higher than that for the $18^{\circ}$ boundary and that they cross over at approximately 5 Gauss. This behavior is based on the self-field generated by the sample, which is dependent on the sample dimensions. As a further test of consistency, we can use the slope of $I_{c}(B)$ to calculate the dimensions of the sample measured and, as noted in the figure, the agreement between this calculated value and the actual dimensions of the sample is within about $25 \%$, supporting the concept that $\mathrm{J}_{\mathrm{c}}$ is determined by flux penetration along the boundary for this orientation.

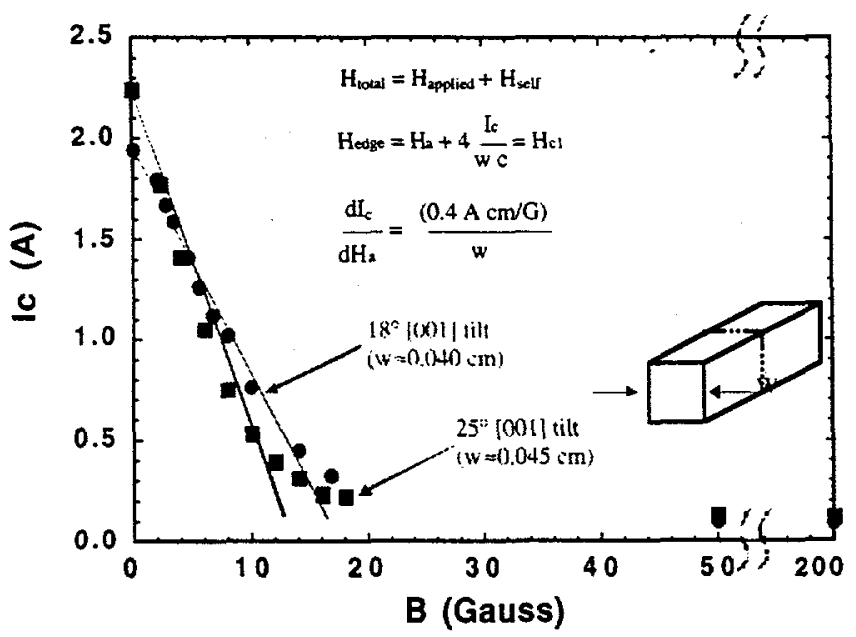

Figure 3. Critical current $\left(I_{c}\right)$ at $77 \mathrm{~K}$ as a function of applied magnetic field. The applied field was parallel to the $c$-axes of each grain.

We have also examined the dependence of the critical current on the orientation of applied magnetic field. In this case, we used a $90^{\circ}$ [100] symmetric tilt boundary. These boundaries are produced in a similar manner to the [001] tilt boundaries discussed to this point. As shown in the inset of Fig. 4 , the applied field $(0.1 \mathrm{~T})$ was rotated in the plane of the boundary. The data of Fig. 4 shows a smooth variation of $I_{c}$ with field orientation, with a maximum corresponding to a field oriented parallel to the $a b$-planes of the grains $\left(\theta \approx 0^{\circ}\right.$, $180^{\circ}$ ) and a minimum when the field is oriented so that it is symmetrically inclined from the $c$-axes of the grains by $45^{\circ}(\theta$ $\approx 90^{\circ}$ ). The absence of any sharp peaks in the curve supports the idea that these planar boundaries are uniform and do not contain discrete pinning centers. However, the general shape of the curve is similar to that measured for single crystals or thin films in which a maximum in $J_{c}$ is measured when the field is oriented along the $a b$-planes and a minimum in $J_{c}$ is measured when the field is parallel to the $c$-axis. Those results are interpreted on the basis on strong pinning of Abrikosov vortices due to the layered structure of YBCO when the field is parallel to $a b$ and relatively weaker pinning when the field is parallel to the $c$-axis. Thus, these results suggest that even though only coreless Josephson vortices are expected along the boundary, pinning can play a role in determining $\mathrm{J}_{\mathrm{s}}$, even for these straight grain boundaries.

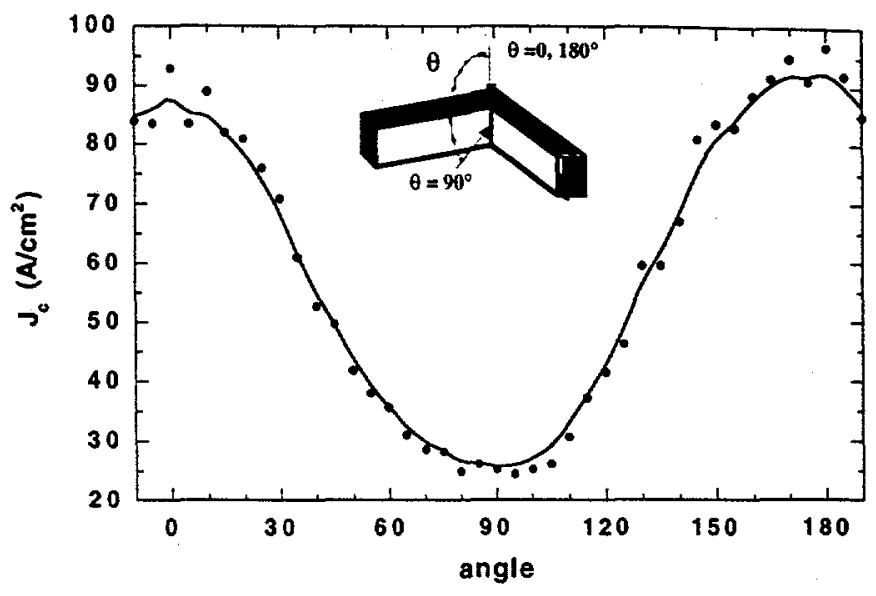

Figure 4. Critical current density (Jc) at $77 \mathrm{~K}$ as a function of applied magnetic field orientation, $\theta$. The applied field of $0.1 \mathrm{~T}$ was oriented in the plane of the boundary as shown in the inset.

\section{IV.DISCUSSION}

All of the data shown is consistent with the conclusion that vortex pinning along a boundary can contribute to the critical current density measured across a boundary. In very low angle grain boundaries, where the vortices may exist as Abrikosov vortices, pinning based on some microstructural features has been shown previously.[11] For the higher angle boundaries considered here, it is Josephson vortices that must be pinned. One aspect of pinning is that the length scale of the pinning center should be of the same order as the characteristic length scale of the vortex. For Josephson vortices, the characteristic length is the Josephson penetration length, $\lambda_{\mathrm{J}}$, which is inversely proportional to the strength of coupling for the junction, and therefore is expected to vary as a function of $\theta$. However, $\lambda_{\mathrm{J}}$ is expected to be rather long for the higher angle boundaries considered here, on the order of a few hundred nanometers to microns. This length scale is consistent with the characteristic period of meanders in thin film boundaries, which would lead to relatively strong pinning in that case. In the bulk grain boundaries, however, there are no features found along the boundary on this length scale and consequently pinning is expected to be weak.

The data of Fig. 4 suggests that the motion of Josephson vortices can be impeded in certain directions, however. The maxima in $J_{c}$ correspond to field orientations for which the vortices must move across the $a b$-planes of the sample. Certain pinning mechanisms cannot be completely excluded as the basis for this behavior due to the geometry of the sample. For example, in this pure symmetric tilt boundary, the dislocations are expected to run parallel to the $a b$-planes, and thus they may contribute to pinning. In addition, the 
Abrikosov vortices in the grains themselves are also more strongly pinning, at least in one direction, and so pinning due to a rigid lattice[12] is also a possibility. However, the dependence of $J_{c}$ on angle can also be explained based on the drag force induced by the intrinsic anisotropy of the YBCO grains, as shown in Fig. 5. The circulating currents that define the Josephson vortex pass through the YBCO grains that consist of superconducting and insulting layers. In an idealized system, the currents will be passed as supercurrent and Josephson current in each of the layers, respectively. In this highly overdamped system, viscous drag is expected to impose a reșistance to motion in the direction of the layering. Note that motion parallel to the layers is not subject to this friction and thus is expected to be free of pinning, as in the case of the [001] tilt boundaries discussed previously.

Finally, it must be pointed out that we consider the presence of vortices and their motion as a key aspect of dissipation. In this respect, we consider only boundaries that are sufficiently long to contain Josephson vortices (longjunction limit). It should be nooted that in many non-device applications, practical conductors as an example, most boundaries are expected to satisfy this conditon. However, the intrinsic conduction across a short length of the boundary is also an essential element. In this model, we do not consider what the precise nature of conduction-across a short length junction is, nor how it varies as a function of $\theta$. However, a number of models based on the structure of the boundary or the pairing symmetry have been proposed that may reasonably explain this dependence.[2-5] This model is distinguished from those in that it assumes some fundamental relationship between the Josephson critical current, $\mathrm{J}_{\mathrm{cj}}$, and $\theta$ and uses that relationship, together with vortex motion, to understand the relationship between the measured critical current, $\mathrm{J}_{\mathrm{c}}$, and $\theta$.

\section{V.CONCLUSION}

The significant differences in the critical current density carried across higher angle [001] tilt grain boundaries in thin films compared to bulk samples can be explained on the basis of pinning of Josephson vortices. A model based on pinning can quantitatively predict the difference in magnitude of $J_{c}$ for equivalent boundaries as well as the difference in the slope of $J_{c}(\theta)$ and the dependence of $I_{c}$ on applied field. A strong basis for pinning is the meandering grain boundary plane typical of thin film bicrystals. In contrast, the straight, highly planar boundaries typical of bulk bicrystals provide very weak pinning. However, it is found that even along these planar boundaries the intrinsic anisotropy of YBCO can lead to pinning of Josephson vortices.

\footnotetext{
The submitted manuscript has been created by the University of Chicago as Operator of Argonne National Laboratory ("Argonne") under Contract No. W-31-109-ENG-38 with the U.S. Department of Energy. The U.S. Government retains for itself, and others acting on its behalf, a paid-up, nonexclusive, irrevocable worldwide license in said article to reproduce, prepare derivative works, distribute copies to the public, and pertorm publicly and display publicly, by or on behalf of the Government.
}

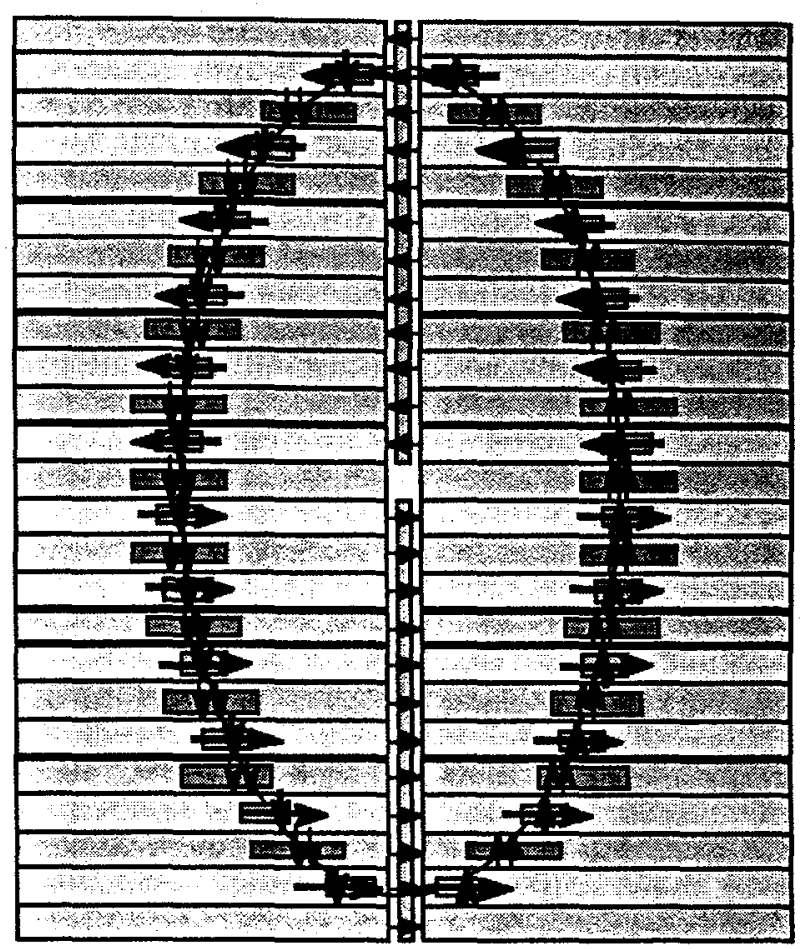

Figure 5. Idealized schematic of a Josephson vortex at a grain boundary in an anisotropic superconductor. The layering in the grains represents superconducting and Josephson components of the curculating currents. As a result of this anisotropy, motion of the vortex along the boundary in or out of the page is not resisted, but motion up or down must overcome a strong drag resistance.

\section{REFERENCES}

1 D. Dimos, P. Chaudhari, J. Mannhart, F.K. LeGoues, Phys. Rev. Lett. 61, 219 (1988).

2 H. Hilgenkamp, J. Mannhart, B. Mayer, Phys. Rev. B53, 14586 (1996).

3 M.F. Chisolm, S.J. Penneyook, Nature 351, 47 (1991).

4 M.B. Field, D.C. Larbalestier, A.S. Parikh, K. Salama, Physica C 280, 221 (1997).

5 N.D. Browning, J.P. Buban, P.D. Nellist, D.P. Norton, M.F. Chisolm, S.J. Pennycook, Physica C 294, 183 (1998).

6 Z.G. Ivanov, P.A. Nilsson, D. Winkler, J.A. Alarco, T. Claeson, E.A. Stepantsov, A.Y. Tzalenchuk, Appl. Phys. Lett. 59, 3030 (1991).

7 N.F. Heinig, R.D. Redwing, I.F. Tsu, A. Gurevich, J.E.

Nordman, S.E. Babcock, D.C. Larbalestier, Appl. Phys. Lett. 69, 577 (1996).

8 V.R. Todt, X.F. Zhang, D.J. Miller, M. St. Louis-Weber, V.P. Dravid, Appl. Phys. Lett. 69, 3746 (1996).

9 K.E. Gray, M.B. Field, D.J. Miller, to appear in Phys. Rev. B (1998).

10 M.B. Field, V.R. Todt, D.J. Miller, D.H. Kim, Appl. Phys. Lett, unpublished.

11 A. Diaz, L. Mechin, P. Berghuis, J.E. Evetts, to appear in Phys. Rev. Lett. (1998).

12 A. Gurevich, DOE-RATF Workshop, July, 1998. 